\title{
Stability for Lie-Trotter products for some operator matrix semigroups
}

\author{
András Bátkai ${ }^{1,2}$, Petra Csomós ${ }^{2,}$, Klaus-Jochen Engel ${ }^{3,}$, and Bálint Farkas ${ }^{2,4, *}$ \\ 1 Eötvös Loránd University, Institute of Mathematics, Pázmány Péter sétány 1/C, 1117 Budapest, Hungary \\ 2 Hungarian Academy of Sciences, Research Group Numerical Analysis and Large Networks, Pázmány Péter sétány 1/C, \\ 1117 Budapest, Hungary \\ ${ }^{3}$ Università degli Studi dell'Aquila, Dipartimento di Ingegneria e Scienze dell'Informazione e Matematica, Via Vetoio \\ (Coppito 1), Stanza 1040, 67100 Coppito (AQ), Italy \\ ${ }^{4}$ Bergische Universität Wuppertal, Fachbereich Mathematik und Informatik, Gaußstraße 20, 42119 Wuppertal, Germany
}

\begin{abstract}
Coupled systems of linear differential equations in Banach spaces can be often handled by the theory of $C_{0}$-semigroups of operator matrices. We study the stability of Lie-Trotter products of such matrix semigroups, and present three classes of examples (abstract delay equations, abstract inhomogeneous equations, abstract dynamic boundary value problems) and some open problems. This survey is based on the papers [1], [2] and [5], to which we refer the interested reader for more details and extensive bibliographical information.
\end{abstract}

Copyright line will be provided by the publisher

The Lie-Trotter product formula provides the motivation and the fundamental background for operator splitting schemes in numerical analysis. Given linear operators $A, B$ on a Banach space $E$ one is interested in the solution of the Cauchy problem $\dot{u}(t)=(A+B) u(t)$ with fixed initial value $u(0)=u_{0}$. The general theory of $C_{0}$-semigroups yields that the problem is well-posed if and only if $A+B$ is the generator of a $C_{0}$-semigroup $(U(t))_{t \geq 0}$, and that in this case the unique solution is given by $u(t)=U(t) u_{0}$ (we refer to standard monographs on semigroup theory, such as [7]). Now suppose that $A, B$ generate the $C_{0}$-semigroups $(S(t))_{t \geq 0},(T(t))_{t \geq 0}$, respectively. If $(\lambda-A-B)$ has dense range and domain $\mathrm{D}(A+B)$, then one has the following equivalence, stating the Lie-Trotter, or sequential splitting, formula:

$$
\text { Convergence: } \quad U(t) x=\lim _{n \rightarrow \infty}\left[S\left(\frac{t}{n}\right) T\left(\frac{t}{n}\right)\right]^{n} x \quad \text { for all } x \in E .
$$

$$
\text { Stability: } \quad \text { There are } M, \omega \geq 0 \text { with }\left\|\left[S\left(\frac{t}{n}\right) T\left(\frac{t}{n}\right)\right]^{n}\right\| \leq M \mathrm{e}^{\omega t} \quad \text { for all } t \geq 0, n \in \mathbb{N} \text {. }
$$

The expressions $\left[S\left(\frac{t}{n}\right) T\left(\frac{t}{n}\right)\right]^{n}$ are called Lie-Trotter products and, as we see, their convergence is equivalent to their stability. This is an instance of the Lax equivalence theorem, and because of this equivalence we shall concentrate on stability issues.

Every $C_{0}$-semigroup $(T(t))_{t \geq 0}$ obeys an exponential estimate of the kind $\|T(t)\| \leq M \mathrm{e}^{\omega t}$ for some $M, \omega \geq 0$, and is called quasicontractive if $M$ can be take to be 1 . If $A, B$ both generate quasicontractive semigroups, then the stability of the corresponding Lie-Trotter products is a simple consequence of the submultiplicativity of the operator norm. In some cases the space $E$ can be renormed such that both semigroups become quasicontractive, in which case the stability is again immediate. This is certainly so if $B \in \mathscr{L}(E)$, i.e., if $B$ is bounded, since we can renorm $E$ such that the semigroup $(S(t))_{t \geq 0}$ generated by $A$ becomes quasicontractive, but a semigroup with bounded generator is quasicontractive.

Proposition 1 (see [1, Prop. 2.4]) Let A be a generator and let B be bounded, then the corresponding Lie-Trotter products are stable.

The importance of splitting procedures becomes inevitable if one considers Cauchy problems (on products $E \times F$ of Banach spaces) given by operator matrices

$$
\dot{x}(t)=\mathcal{A} x(t), \quad \mathcal{A}: \mathrm{D}(\mathcal{A}) \subseteq E \times F \rightarrow E \times F, \quad \text { where formally } \quad \mathcal{A}=\left(\begin{array}{ll}
A & D \\
C & B
\end{array}\right) .
$$

In general, $\mathcal{A}$ can be represented only formally as an operator matrix: Its domain is usually nondiagonal (i.e., contains a certain coupling of the two coordinates). That is why, even if $C=0$ and $D=0$ the semigroup generated by $\mathcal{A}$ may not be of diagonal but rather of upper triangular form. Here is a characterization of such matrix semigroups:

Proposition 2 (see [1, Prop. 2.1]) Let $\mathcal{A}$ generate the semigroup $(\mathcal{T}(t))_{t \geq 0}$ on $E \times F$. Define $A: \mathrm{D}(A) \rightarrow E$ by

$$
A x:=\pi_{1}\left(\mathcal{A}\left(\begin{array}{l}
x \\
0
\end{array}\right)\right) \text { for } x \in \mathrm{D}(A):=\left\{z \in E:\left(\begin{array}{l}
z \\
0
\end{array}\right) \in \mathrm{D}(\mathcal{A})\right\},
$$

where $\pi_{i}$ denotes the projection onto the $i^{\text {th }}$ coordinate. Then the semigroup has upper triangular form, i.e.

$$
\mathcal{T}(t)=\left(\begin{array}{cc}
S(t) & R(t) \\
0 & T(t)
\end{array}\right) \quad \text { for all } t \geq 0,
$$

if and only if the following two conditions are satisfied:

\footnotetext{
* Corresponding author: e-mail farkas@uni-wuppertal.de, phone +49 202 162637,
} 
(i) For all $x \in E$ with $\left(\begin{array}{l}x \\ 0\end{array}\right) \in \mathrm{D}(\mathcal{A})$ we have $\pi_{2}\left(\mathcal{A}\left(\begin{array}{l}x \\ 0\end{array}\right)\right)=0$.

(ii) There exists $\lambda \in \rho(A)$ satisfying $\Re \lambda>s(\mathcal{A})$ (the spectral bound of $\mathcal{A}$ ).

Moreover, in this case $(S(t))_{t \geq 0}$ and $(T(t))_{t \geq 0}$ are $C_{0}$-semigroups and the generator of $(S(t))_{t \geq 0}$ is $A$.

For upper triangular matrix semigroups we have the following stability result:

Proposition 3 ( $\left[1\right.$, Thm. 2.2]) Suppose that $\mathcal{A}_{1}$ and $\mathcal{A}_{2}$ generate on $E \times F$ the $C_{0}$-semigroups $\left(\mathcal{T}_{1}(t)\right)_{t \geq 0}$ and $\left(\mathcal{T}_{2}(t)\right)_{t \geq 0}$ that are of upper triangular form

$$
\mathcal{T}_{1}(t)=\left(\begin{array}{cc}
S_{1}(t) & R_{1}(t) \\
0 & T_{1}(t)
\end{array}\right) \quad \text { and } \quad \mathcal{T}_{2}(t)=\left(\begin{array}{cc}
S_{2}(t) & R_{2}(t) \\
0 & T_{2}(t)
\end{array}\right)
$$

and suppose that there exist $M, \omega \geq 0$ such that

$$
\left\|R_{1}(t)\right\| \leq M t \mathrm{e}^{\omega t} \quad \text { and } \quad\left\|R_{2}(t)\right\| \leq M t \mathrm{e}^{\omega t} \quad \text { for } t \geq 0 .
$$

Then the Lie-Trotter products of $\left(\mathcal{T}_{1}(t)\right)_{t \geq 0}$ and $\left(\mathcal{T}_{2}(t)\right)_{t \geq 0}$ are stable if and only if the Lie-Trotter products of $\left(S_{1}(t)\right)_{t \geq 0}$ and $\left(S_{2}(t)\right)_{t \geq 0}$ and the Lie-Trotter products of $\left(T_{1}(t)\right)_{t \geq 0}$ and $\left(T_{2}(t)\right)_{t \geq 0}$ are stable.

The next result characterizes those triangular matrix semigroups for which the foregoing stability result applies. For the definition of the Favard spaces $\operatorname{Fav}_{0}(A), \operatorname{Fav}_{1}(A)$ and the extrapolated semigroups we refer to [7, Sec. II.5]. In the situation of Proposition 2 for an upper triangular semigroup $(\mathcal{T}(t))_{t>0}$ of the form (1) we denote by $A$ and $B$ the generators of the diagonal entries and set $\mathcal{D}=\left(\begin{array}{ll}A & 0 \\ 0 & B\end{array}\right)$ with diagonal domain, which then generates the diagonal semigroup on $E \times F$.

Proposition 4 (see [1, Prop. 2.6]) Let $(\mathcal{T}(t))_{t \geq 0}$ be a triangular semigroup of the form (1) on the product space $E \times F$ with generator $\mathcal{A}$. Then the following assertions are equivalent:

(i) There exist $M, \omega \geq 0$ such that $\|R(t)\| \leq M t \mathrm{e}^{\omega t}$ for all $t \geq 0$.

(ii) There exists $P: F \rightarrow \operatorname{Fav}_{0}(A)$ bounded operator such that $\mathcal{A}=\left.\left(\mathcal{D}_{-1}+\mathcal{P}\right)\right|_{E \times F}$ where $\mathcal{P}=\left(\begin{array}{cc}0 & P \\ 0 & 0\end{array}\right)$.

This result implies that for reflexive spaces $E, F$ we are just in the situation of Proposition 1, i.e., when the upper triangular semigroup is a bounded perturbation of the diagonal one, cf. [7, Sec. III.3].

\section{Delay equations}

Consider the delay equation

$$
\begin{cases}\dot{u}(t)=B u(t)+\Phi u_{t}, & t \geq 0 \\ u(s)=f(s), & s \in[-1,0]\end{cases}
$$

on a Hilbert space $H$, where $B: \mathrm{D}(B) \subseteq H \rightarrow H$ is linear, $f:[-1,0] \rightarrow H$ is a given initial function, $u_{t}:[-1,0] \rightarrow H$ is the history function defined by $u_{t}(s)=u(t+s)$ for $s \in[-t, 0]$ and $u_{t}(s)=f(t+s)$ for $s \in[-1,-t)$, and where $\Phi$ is a linear operator, called the delay operator, mapping $H$-valued functions on $[-1,0]$ to $H$. Typical choices for the delay operator are (a) the point delays, e.g., $\Phi v=v(-1)$ or (b) distributed delays, e.g., $\Phi v=\int_{-1}^{-0.5} v(\sigma) \mathrm{d} \sigma$, or (c) (as a matter of fact, including both (a) and (b)) $\Phi v=\int_{-1}^{0} v(\sigma) \mathrm{d} \eta(\sigma)$ with $\eta \in \mathrm{BV}([-1,0] ; \mathscr{L}(H))$.

By introducing a new unknown function $x(t):=\left(\begin{array}{c}u_{t} \\ u(t)\end{array}\right)$ the delay equation can be recast as an abstract Cauchy problem on the product space $\mathrm{L}^{p}([-1,0] ; H) \times H, p \in[1, \infty)$ as

$$
\left\{\begin{array}{l}
\dot{x}(t)=\mathcal{A} x(t) \\
x(0)=\left(\begin{array}{l}
f \\
x
\end{array}\right)
\end{array} \quad \text { with } \quad \mathcal{A}:=\left(\begin{array}{cc}
\frac{\mathrm{d}}{\mathrm{d} \sigma} & 0 \\
\Phi & B
\end{array}\right) \quad \text { and } \quad \mathrm{D}(\mathcal{A}):=\left\{\left(\begin{array}{l}
f \\
x
\end{array}\right) \in \mathrm{W}^{1, p}([-1,0] ; H) \times \mathrm{D}(B): f(0)=x\right\},\right.
$$

where $\frac{\mathrm{d}}{\mathrm{d} \sigma}$ stands for the first derivative with domain $\mathrm{W}^{1, p}([-1,0] ; H)$. The delay equation is well-posed if and only if $\mathcal{A}$ generates a $C_{0}$-semigroup in which case the solution $u$ is given by $\pi_{2} \circ x$, see [3] and [9]. This is so if $B$ is a generator and $\Phi$ is any of the forms (a)-(c) above. In this setting it is natural to apply splitting to separate the delayed part from the undelayed one. Depending on the nature of the delay two choices are possible (the coupling is incorporated in the domain of the operator containing $\left.\frac{\mathrm{d}}{\mathrm{d} \sigma}\right)$ :

(a) For point delays (or for delay of the general class (c)) we can split

$$
\left(\begin{array}{ll}
\frac{\mathrm{d}}{\mathrm{d} \sigma} & 0 \\
\Phi & B
\end{array}\right)=\left(\begin{array}{cc}
\frac{\mathrm{d}}{\mathrm{d} \sigma} & 0 \\
\Phi & 0
\end{array}\right)+\left(\begin{array}{ll}
0 & 0 \\
0 & B
\end{array}\right) .
$$

In this way we can separate the delay part, and we only need to solve a delayed ordinary differential equation and a decoupled abstract Cauchy problem. The corresponding Lie-Trotter products are stable because the space can be renormed, if the delay operator contains the point evaluation at -1 , such that both semigroups become contractive, see [9], and also [2]. 
(b) For distributed delays (continuous delay operator) we can split

$$
\left(\begin{array}{cc}
\frac{\mathrm{d}}{\mathrm{d} \sigma} & 0 \\
\Phi & B
\end{array}\right)=\left(\begin{array}{cc}
\frac{\mathrm{d}}{\mathrm{d} \sigma} & 0 \\
0 & B
\end{array}\right)+\left(\begin{array}{cc}
0 & 0 \\
\Phi & 0
\end{array}\right)
$$

which has the advantage that the semigroups generated by the two operators have particularly simple structure. The Lie-Trotter products are stable because one of the generators is bounded, see Proposition 1, and also [5].

\section{Inhomogeneous Abstract Cauchy Problems}

Consider the inhomogeneous Cauchy problem

$$
\left\{\begin{array}{l}
\dot{u}(t)=A u(t)+f(t), \quad t \geq 0, \\
u(0)=u_{0}
\end{array}\right.
$$

for a linear operator $A$ on a Banach space $E$. A standard method to is to rewrite the inhomogeneous equation as a homogeneous one the product space $E \times F$, where $F=F\left(\mathbb{R}_{+} ; E\right)$ denotes a Banach space of $E$-valued functions defined on $\mathbb{R}_{+}$on which the left-shift semigroup $(S(t))_{t \geq 0}$ is strongly continuous. Consider

$$
\mathcal{A}=\left(\begin{array}{cc}
A & \delta_{0} \\
0 & \frac{d}{\mathrm{~d} s}
\end{array}\right) \quad \text { with diagonal domain } \quad \mathrm{D}(\mathcal{A})=\mathrm{D}(A) \times F_{1}\left(\mathbb{R}_{+} ; E\right)
$$

where $F_{1}:=F_{1}\left(\mathbb{R}_{+} ; E\right)$ is the domain of the generator of the left-shift semigroup, and $\delta_{0}(v):=v(0)$ is the point evaluation at 0 . By [7, Sec. VI.7] for the choices $F\left(\mathbb{R}_{+} ; E\right)=\mathrm{C}_{0}\left(\mathbb{R}_{+} ; E\right)$ or $\mathrm{L}^{p}\left(\mathbb{R}_{+} ; E\right)(p \in[1, \infty))$ we have $F_{1}=\mathrm{C}_{0}^{1}\left(\mathbb{R}_{+} ; E\right)$ and $F_{1}=\mathrm{W}^{1, p}\left(\mathbb{R}_{+} ; E\right)$, respectively, and the inhomogeneous equation (iACP) is equivalent to the abstract Cauchy problem

$$
\left\{\begin{array}{l}
\dot{x}(t)=\mathcal{A} x(t), \quad t \geq 0, \\
x(0)=\left(\begin{array}{c}
u_{0} \\
f
\end{array}\right),
\end{array}\right.
$$

with the new unknown function $x(t)=(u(t), x(t))$. If $A$ is a generator then so is $\mathcal{A}$ and the solution $u(t)$ is given by $u=\pi_{1} \circ x$. We apply splitting to (iACP):

$$
\left\{\begin{array}{l}
\dot{u}(t)=\left(A_{1}+A_{2}\right) u(t)+\left(f_{1}+f_{2}\right)(t), \quad t \geq 0, \\
u(0)=u_{0} .
\end{array}\right.
$$

and obtain two inhomogeneous equations $\dot{v}_{1}(t)=A_{1} v_{1}(t)+f_{1}(t)$ and $\dot{v}_{2}(t)=A_{2} v_{2}(t)+f_{2}(t)$. By rewriting these problems on the product space $E \times F \times F$ we arrive at

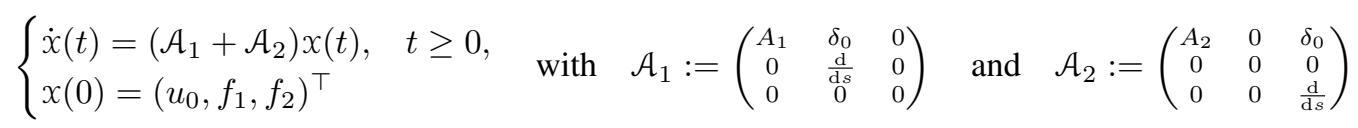

with respective domains $\mathrm{D}\left(A_{1}\right) \times F_{1} \times F$ and $\mathrm{D}\left(A_{2}\right) \times F \times F_{1}$, and new unknown function $x(t)=(u(t), x(t), y(t))$.

Proposition 1 (see [1, Prop. 3.1, Prop. 3.4]) In either of the cases $F=\mathrm{C}_{0}\left(\mathbb{R}_{+} ; E\right)$ or $F=\mathrm{L}^{p}\left(\mathbb{R}_{+} ; E\right)$ the Lie-Trotter products of the semigroups generated by $\mathcal{A}_{1}$ and $\mathcal{A}_{2}$ are stable if and only if the Lie-Trotter products for the semigroups generated by $A_{1}$ and $A_{2}$ are stable.

The case when $F=\mathrm{C}_{0}\left(\mathbb{R}_{+} ; E\right)$ follows by an application of Proposition 1, the other case is proved by direct calculation exploiting special properties of the norm on $\mathrm{L}^{p}$. Informally, the previous proposition means that the sequential splitting for the inhomogeneous problem is stable if and only if it is stable for the homogeneous one.

\section{Abstract Boundary Feedback Systems}

Consider the following problem

$$
\begin{cases}\dot{x}(t)=A_{m} x(t), & t \geq 0, \\ \dot{y}(t)=B y(t)+C x(t), & t \geq 0, \\ L x(t)=y(t), & t \geq 0, \\ x(0)=x_{0}, y(0)=y_{0}, & \end{cases}
$$

called abstract boundary feedback system, where the unknown functions $x:[0, \infty) \rightarrow E, y:[0, \infty) \rightarrow F$ have values in the Banach spaces $E, F$, the operators $A_{m}, B$ act on $E$ and $F$, respectively, and $L: E \rightarrow F$ is linear. The operator $A_{m}$ is an 
operator with a large (maximal) domain (e.g., $A_{m}$ is differential operator without boundary conditions) and is typically not a generator. This enlargment of the domain is neccessary because of the coupling condition $L x=y$. Interpreting the dynamics given by $A_{m}$ as interior dynamics and the one corresponding to $B$ as the boundary dynamics, where the coupling is given by $L x=y$, the operator $C$ may be thought of as a feedback effect from the interior to the boundary, see Example 3 below for an explanation. In [4, Sec. 2] it is shown that under next set of conditions the problem can be equivalently formulated as an abstract Cauchy problem on the product space $E \times F$ :

Assumption 1 (i) $A:=\left.A_{m}\right|_{\text {ker } L}$ is a generator on $E$. (ii) $L: \mathrm{D}\left(A_{m}\right) \rightarrow F$ is surjective. (iii) $\left(\begin{array}{c}A_{m} \\ L\end{array}\right): \mathrm{D}\left(A_{m}\right) \rightarrow E \times F$ is closed. (iv) $B$ is a generator on $F$.

More precisely, to study well-posedness of (ABFS) one needs to study the generator property of the operator

$$
\mathcal{A}_{C}:=\left(\begin{array}{cc}
A_{m} & 0 \\
C & B
\end{array}\right) \quad \text { with domain } \quad \mathrm{D}\left(\mathcal{A}_{C}\right):=\left\{\left(\begin{array}{l}
x \\
y
\end{array}\right) \in \mathrm{D}\left(A_{m}\right) \times \mathrm{D}(B): L x=y\right\} .
$$

Example 2 Let $H$ be a Hilbert space, $E=\mathrm{L}^{p}([-1,0] ; H)(p \in[1, \infty))$, let $A_{m}=\frac{\mathrm{d}}{\mathrm{d} \sigma}$ be the first derivative with domain $\mathrm{D}\left(A_{m}\right)=\mathrm{W}^{1, p}([-1,0] ; H), L: \mathrm{W}^{1, p}([-1,0] ; H)$ the trace at $0, L f=f(0)$, let $B$ be the generator of a $C_{0}$-semigroup on $F=H$, and $C f=\Phi f=\int_{-1}^{0} f(s) \mathrm{d} \eta(s)$ as in Section 1. Then $A=\left.A_{m}\right|_{\text {ker } L}$ is the generator of the nilpotent left-shift on $\mathrm{L}^{p}([-1,0] ; H)$ and $\mathcal{A}_{C}$ is a generator. We see that delay equations are included in this setting.

Example 3 (see [4]) Let $\Omega$ be a bounded domain with smooth boundary. Let $E=\mathrm{L}^{2}(\Omega), F=\mathrm{L}^{2}(\partial \Omega), A_{m}=\Delta_{\Omega}$ the maximal Laplace operator, $\mathrm{D}\left(A_{m}\right)=\left\{f \in \mathrm{H}^{\frac{1}{2}}(\Omega) \cap \mathrm{H}_{l o c}^{2}(\Omega): \Delta f \in \mathrm{L}^{2}(\Omega)\right\}, L=$ the trace operator, $B=\Delta_{\partial \Omega}-\mu, \Delta_{\partial \Omega}$ the Laplace-Beltrami operator. The operator $C$ may describe a feedback (e.g., cooling) from the heat transfer inside $\Omega$ to the boundary $\partial \Omega$. In [4] it is shown that for bounded $C$ the problem is well-posed.

We consider first the situation without feedback, i.e., when $C=0$. We apply splitting to separate the interior and boundary dynamics and consider $\mathcal{A}_{0}=\mathcal{A}_{1}+\mathcal{A}_{2}$ for

$$
\mathcal{A}_{1}:=\left(\begin{array}{cc}
A_{m} & 0 \\
0 & 0
\end{array}\right), \quad \mathrm{D}\left(\mathcal{A}_{1}\right):=\left\{\left(\begin{array}{l}
x \\
y
\end{array}\right) \in \mathrm{D}\left(A_{m}\right) \times F: L x=y\right\}, \quad \mathcal{A}_{2}:=\left(\begin{array}{ll}
0 & 0 \\
0 & B
\end{array}\right), \quad \mathrm{D}\left(\mathcal{A}_{2}\right):=E \times \mathrm{D}(B) .
$$

Then by [4, Cor. 2.9] the operator $\mathcal{A}_{1}$ generates a semigroup, which is upper triangular by Proposition 2. The operator $\mathcal{A}_{2}$ evidently generates a diagonal semigroup.

Proposition 4 (see [1, Prop. 3.9]) Suppose that Assumptions 1 are satisfied, $0 \in \rho(A)$, and $C=0$. If $\mathrm{D}\left(A_{m}\right) \subset \mathrm{Fav} 1(A)$, then the Lie-Trotter products of the semigroup generated by $\mathcal{A}_{1}$ and $\mathcal{A}_{2}$ are stable.

The proof relies on Proposition 3 and on a result of Desch and Schappacher about perturbations of semigroups, see [6]. If we add a bounded feedback to the problem (ABFS), i.e., $C \neq 0$, by Proposition 1 we obtain the next result:

Proposition 5 (see [1, Prop. 3.10]) Let $\mathrm{C}:=\left(\begin{array}{cc}0 & 0 \\ C & 0\end{array}\right)$ for some $C \in \mathscr{L}(E, F)$. Then Lie-Trotter products of the semigroups generated by $\mathcal{A}_{0}$ and $\mathrm{C}$ are stable.

Remarks and problems: 1. The results presented in the preceding are valid for the Strang and for the weighted splittings, see [1]. What about other types of splittings?

2. Can one relax, or under which conditions, the growth assumption $O(1)$ near $t=0$ in Proposition 3 ?

3. What about nontriangular matrices? Can one give abstract but applicable neccessary conditions for the stability of the corresponding Lie-Trotter products?

4. Second order problems can also be handled by operator matrix semigroups. What about the stability of Lie-Trotter products in this case? In [8] there are already some partial results by S. Orzlowski.

5. Can one put the $L^{p}$-result for inhomogeneous equations in a more general abstract setting?

6. Proposition 4 is not applicable for Example 3. Are the Lie-Trotter products as in Proposition 4 stable for this example? Can one put this in a more abstract framework?

\section{References}

[1] A. Bátkai,P. Csomós, K.-J. Engel, B. Farkas, Stability and convergence of product formulas for operator matrices, Int. Eq. Op. Th. 74, 281-299 (2012).

[2] A. Bátkai, P. Csomós, B. Farkas, Operator splitting for dissipative delay equations, http://arxiv.org/abs/1009.1981.

[3] A. Bátkai, S. Piazzera, Semigroups for Delay Equations, (A K Peters, Wellesley, Massachusetts, 2005).

[4] V. Casarino, K.-J. Engel, R. Nagel, and G. Nickel, A semigroup approach to boundary feedback systems, Int. Eq. Op. Th. 47, 289-306 (2003).

[5] P. Csomós and G. Nickel, Operator splitting for delay equations, Comput. Math. Appl. 55, 2234-2246 (2008).

[6] W. Desch and W. Schappacher, Some generation results for perturbed semigroups, Lect. Notes in Pure and Appl. Math., vol. 116, (Marcel Dekker, 1989), pp. 125-152.

[7] K.-J. Engel and R. Nagel, One-Parameter Semigroups for Linear Evolution Equations, Graduate Texts in Math. vol. 194 (SpringerVerlag, New York, Heidelberg, Berlin, 2000).

[8] S. Orzlowski, Operator Splitting für abstrakte Cauchy Probleme zweiter Ordnung, MSc Thesis (Universität Siegen, 2013).

[9] G. F. Webb, Functional differential equations and nonlinear semigroups in $L^{p}$-spaces, J. Diff. Eq. 20, 71-89 (1976). 